\title{
Avaliação do módulo de elasticidade de peças de madeira laminada colada (MLC) obtido por meio do ensaio de vibração transversal
}

\author{
Evaluation of the modulus of elasticity of glued \\ laminated timber beams obtained by transverse vibration
}

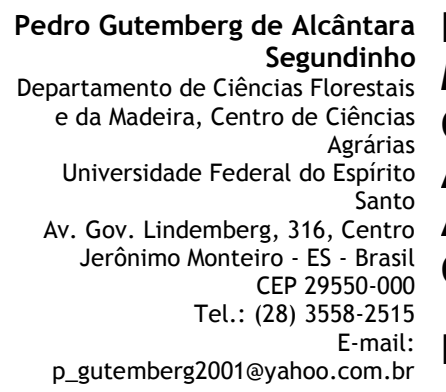

Marcelo Rodrigo Carreira Coordenação de Engenharia Civi Universidade Tecnológica Federal do

BR $369, \mathrm{~km} 0,5$, Campus Campo Mourão

Campo Mourão - PR - Brasil CEP 87301-005

Tel.: (44) 3523-4156

E-mail: carreira@utfpr.edu.br

Carlito Calil Neto Departamento de Engenharia de Estruturas, Escola de Engenharia de

São Carlos

Universidade de São Paulo Av. Trabalhador Sãocarlense, 400 Parque Arnold Schimidt São Carlos - SP - Brasil CEP 13566-590

Tel.: (16) 3373-9483 E-mail: netousp@gmail.com

Adair José Regazzi Departamento de Ciências Florestais e da Madeira, Centro de Ciências Agrárias Universidade Federal do Espírito

E-mail: adairreg@ufv.br

Antonio Alves Dias Departamento de Engenharia de Estruturas, Escola de Engenharia de São Carlos

Universidade de São Paulo Tel.: (16) 3373-8207

E-mail: dias@sc.usp.br

Carlito Calil Junior Departamento de Engenharia de Estruturas, Escola de Engenharia de São Carlos, Universidade de São Paulo Tel.: (16) 3373-9320

E-mail: calil@sc.usp.br

Recebido em 21/04/10/12

Aceito em 16/04/13

\section{Pedro Gutemberg de Alcântara Segundinho Marcelo Rodrigo Carreira \\ Carlito Calil Neto \\ Adair José Regazzi \\ Antonio Alves Dias \\ Carlito Calil Junior \\ Resumo}

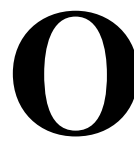

controle da qualidade é fundamental no processo de fabricação de peças de madeira laminada colada (MLC), e uma das formas desse controle pode ser por meio da avaliação não destrutiva do módulo de elasticidade. O objetivo deste trabalho foi comparar os valores de módulo de elasticidade das peças de MLC obtidos por meio do ensaio de vibração transversal livre e do ensaio de flexão estática, ambos feitos antes e após tratamento preservativo (contra a ação de agentes biológicos), realizado pelo processo de Célula Cheia. No total, foram produzidas 54 peças de MLC de dimensões nominais de $9 \mathrm{~cm}$ x $9 \mathrm{~cm}$ x $200 \mathrm{~cm}$, com três espécies de madeira de reflorestamento coladas com dois adesivos de aplicação estrutural e tratadas com três preservantes. Foi obtida uma correlação significativa entre os valores do módulo de elasticidade obtidos pelos dois métodos ( $\mathrm{r}$ variou entre 0,85 e 0,99 ). Concluiu-se que o ensaio de vibração transversal livre pode ser utilizado para avaliação do módulo de elasticidade de peças de MLC, sendo uma alternativa ao ensaio de flexão estática.

Palavras-chave: Madeira laminada colada. Avaliação não destrutiva. Vibração transversal.

\section{Abstract}

Quality control is essential in the manufacturing process of glued-laminated (glulam) timber elements. This control can be done by nondestructive evaluation of the modulus of elasticity. The purpose of this study is to compare the values of modulus of elasticity of glulam elements obtained by free transverse vibration tests and static bending, before and after preservative treatment, for protection against the action of biological agents, performed by the full cell process. A total of 54 glulam elements were produced, with nominal sizes of $9 \mathrm{~cm} \times 9 \mathrm{~cm} \times 200 \mathrm{~cm}$, with three species of reforestation wood, glued with two structural application adhesives and treated with three preservatives. A good correlation between the values of modulus of elasticity was obtained ( $r$ ranges between 0.85 and 0.99 ). The conclusion was made that the free vibration test is useful for evaluating the modulus of elasticity of glulam elements, and it is an alternative to the static bending test.

Keywords: Glued laminated timber. Nondestructive evaluation. Transverse vibration. 


\section{Introdução}

A madeira laminada colada (MLC) é um produto de engenharia utilizado largamente na Europa, nos Estados Unidos e no Canadá, porque pode ser produzido a partir de praticamente todas as espécies de madeira e mesmo daquelas obtidas de árvores jovens e de pequeno diâmetro. A dependência da madeira de árvores com maior idade, consideradas necessárias para fornecer uma alta resistência e grande envergadura para o projeto arquitetônico, atualmente não é viável (YANG et al., 2008). Assim, uma de suas maiores vantagens é aproveitar uma grande quantidade de peças com pequenas dimensões, por se tratar de produto obtido pela associação de peças da madeira (lâminas) que requer precisão de fabricação em todos os seus estágios. As lâminas, unidas por colagem, ficam dispostas de modo que suas fibras estejam paralelas entre si (BODIG; JAYNE, 1993) e formem um componente estrutural. Tais lâminas de madeira são selecionadas e coladas com adesivo à prova d'água, sob pressão variável de 0,7 a 1,5 MPa. Outro fator importante é a utilização qualitativa de peças de madeira de primeira categoria, conforme especificado pela norma brasileira NBR 7190 (ABNT, 1997), usando as de maior classe de resistência nas regiões mais solicitadas. Pode-se também variar a espécie de madeira na estrutura, de acordo com a solicitação de resistência das laminações em cada região da viga (FOREST ..., 1999).

Apesar do rigoroso controle sobre as matériasprimas e o processo de produção, os elementos estruturais de MLC apresentam certa variabilidade nas propriedades mecânicas inerentes ao próprio material empregado. Assim sendo, é fundamental verificar se as propriedades mecânicas definidas em projeto foram atendidas. Segundo CAN/CSA 0177 (CANADIAN..., 2006), o produto acabado pode ser testado em condições laboratoriais; portanto, é necessário o controle de qualidade na produção para assegurar que as propriedades físicas e mecânicas da MLC sejam adequadas àquelas especificadas nas normas vigentes. É possível realizar uma caracterização eficaz por meio de métodos não destrutivos, pois a avaliação pode ser realizada no próprio elemento, sem a necessidade de extração de corpos de prova (ROSS; PELLERIN, 1994). Podem ser empregados ensaios não destrutivos de flexão estática, pelos quais é possível estimar o módulo de elasticidade à flexão dos elementos (AMERICAN..., 1997). Dependendo das dimensões dos elementos estruturais, é necessário dispor de um sistema de reação de grandes proporções. O módulo de elasticidade dos elementos também pode ser obtido por meio de ensaios dinâmicos não destrutivos, cujos aparelhos utilizam acelerômetros ou microfones e medem a frequência de vibração da amostra de madeira (MOCHAN; CONNOLLY; MOORE, 2009). Os ensaios não destrutivos podem ser realizados por meio de equipamentos de menores custos e dimensões em relação ao sistema necessário para a realização de ensaios de flexão estática (CARREIRA et al., 2012).

O objetivo deste trabalho foi avaliar o módulo de elasticidade de peças de MLC por meio de vibração transversal, antes e depois do tratamento preservativo, produzidas a partir de três espécies de madeira de reflorestamento e com dois adesivos estruturais. Nesse estudo, foi comparada a técnica de Avaliação Não Destrutiva (AND) de vibração transversal livre com o ensaio estático padronizado na norma brasileira NBR 8458 (ABNT, 2010).

\section{Materiais e métodos}

Nesse item estão descritos a fabricação das peças de MLC, o ensaio de flexão estática, o ensaio de vibração transversal livre e as análises estatísticas. Tanto a fabricação quanto os ensaios foram realizados no Laboratório de Madeiras e de Estruturas de Madeira (LaMEM), do Departamento de Engenharia de Estruturas (SET), da Escola de Engenharia de São Carlos (EESC), da Universidade de São Paulo (USP).

\section{Materiais}

Foram produzidas peças de MLC com madeira de Teca (Tectona grandis), Pinus oocarpa (Pinus oocarpa shiede) e Lyptus ${ }^{\circledR}$, marca registrada de madeira de reflorestamento desenvolvida com cruzamento de árvores selecionadas, cuja detentora da marca é a Bahia Produtos de Madeira, joint venture formada pelas empresas Fibria Celulose S.A. e Weyerhaeuser NR Company. Para cada espécie foram produzidas 18 peças de MLC, sendo 9 coladas com o adesivo à base de resorcina fenol formaldeído (RFF) e 9 com o adesivo à base de poliuretano (PUR), totalizando 54 peças para as três espécies de madeira. As peças de MLC foram montadas com lâminas classificadas visual e mecanicamente, com distribuição qualitativa, posicionando as de melhor qualidade nas partes extremas da seção transversal. Elas foram coladas com linha de cola dupla (gramatura de 300 a 350 $\mathrm{g} / \mathrm{m}^{2}$ ) e pressão de $1 \mathrm{MPa}$. Tais peças de MLC tinham comprimento de $200 \mathrm{~cm}$, seção transversal de $9 \mathrm{~cm} \times 9 \mathrm{~cm}$ e foram confeccionadas com três peças de madeira (Figura 1b), cada lâmina com 3

8 Segundinho, P. G. de A.; Carreira, M. R.; Calil Neto, C.; Regazzi, A. J.; Dias, A. A.; Calil Junior, C. 
cm de espessura. A Figura 1a ilustra a fabricação de dez peças de MLC em uma prensa do tipo " $A$ ".

As peças de MLC foram confeccionadas sem tratamento preservativo e em seguida passaram pelos ensaios de flexão estática e vibração transversal livre. $\mathrm{Na}$ sequência, elas foram submetidas a tratamento preservativo sob pressão pelo método Bethel - processo de Célula Cheia com as substâncias hidrossolúveis arseniato de cobre cromatado (CCA) e borato de cobre cromatado $(\mathrm{CCB})$, com base salina ou óxida. Decorrido o tempo de secagem, as peças de MLC foram novamente submetidas aos ensaios de flexão estática e vibração transversal livre. Em cada grupo de 9 peças de MLC de uma mesma espécie de madeira com o mesmo adesivo de colagem, foram tratadas 4 CCA, 3 CCB-óxido e 2 CCBsalino. Os três tipos de preservativos foram empregados na concentração $2 \%$ de ingredientes ativos.

\section{Ensaio de flexão estática}

O ensaio de flexão estática das peças de MLC foi realizado de acordo com a norma brasileira NBR 8458 (2010). A viga foi apoiada no centro do vão e aplicaram-se forças iguais situadas a $10 \mathrm{~cm}$ das extremidades (Figura 2). As flechas foram medidas nos pontos de aplicação dessas forças.

O módulo de elasticidade estático $\left(E_{\mathrm{M}}\right)$ foi obtido com o uso da Equação 1, avaliada com os valores médios de deslocamentos e esforços.

$E_{\mathrm{M}}=\frac{\mathrm{P} \cdot \mathrm{a}^{3}}{3 \cdot \mathrm{I} \cdot \delta}$,
Em que:

$\mathrm{P}=4 \mathrm{kN}$ (valor de cada força);

$\mathrm{a}=$ distância entre o ponto de aplicação de cada força até o centro do vão (m);

$\delta=$ flechas medidas nos pontos de aplicação das forças (m); e

$\mathrm{I}=$ momento de inércia da seção transversal $\left(\mathrm{m}^{4}\right)$.

\section{Ensaio de vibração transversal livre}

O ensaio de vibração transversal livre foi realizado adotando-se a condição de contorno livre-livre (MCCONNELL; VAROTO, 2008). Para tanto, as peças foram suspensas utilizando-se linhas de nylon e molas de pequena rigidez, conforme mostrado no esquema de ensaio nas Figuras 3(a) e 3(b). A linha de nylon foi posicionada nos pontos nodais do primeiro modo de flexão de uma viga em suspensão livre-livre. O módulo de elasticidade dinâmico é obtido por meio da Equação 2.

$$
E_{\mathrm{vt}}=\frac{\mathrm{f}^{2} \cdot(2 \cdot \pi)^{2} \cdot \mathrm{M} \cdot \mathrm{L}^{3}}{(\lambda)^{4} \cdot \mathrm{I}},
$$

Em que:

$\mathrm{f}=$ frequência do primeiro modo de flexão $(\mathrm{Hz})$;

$\mathrm{M}=$ massa da viga $(\mathrm{kg})$;

$\mathrm{L}=$ comprimento $(\mathrm{m})$;

$\lambda=$ constante para a condição de contorno livrelivre (4,73); e

Eq. $1 \quad \mathrm{I}=$ momento de inércia da seção transversal $\left(\mathrm{m}^{4}\right)$.

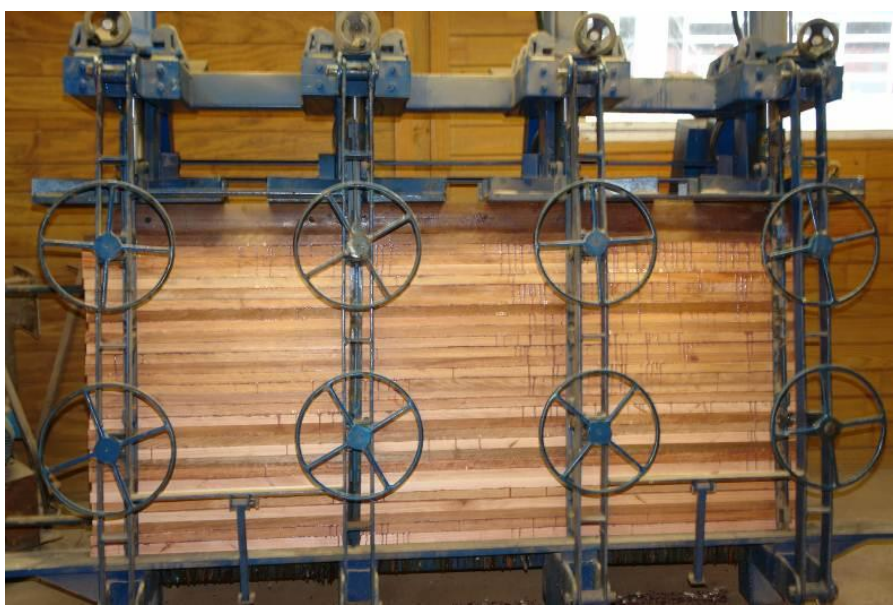

(a)

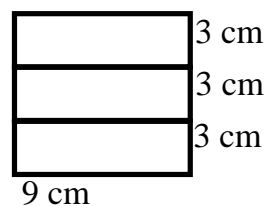

(b) Seção

Figura 1 - Fabricação (a) e seção transversal e (b) das peças de MLC na prensa do tipo "A" 


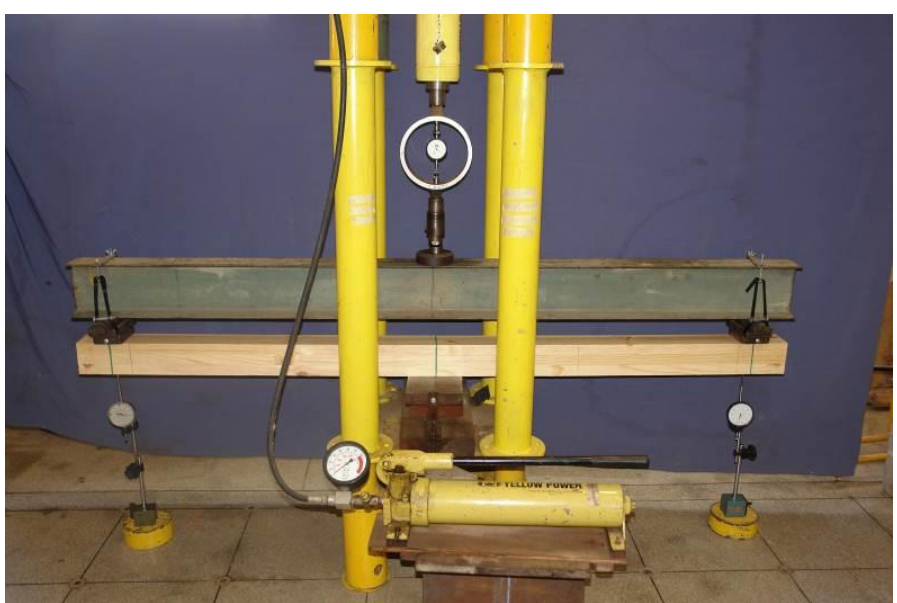

Figura 2 - Ensaio de flexão estática nas peças de MLC

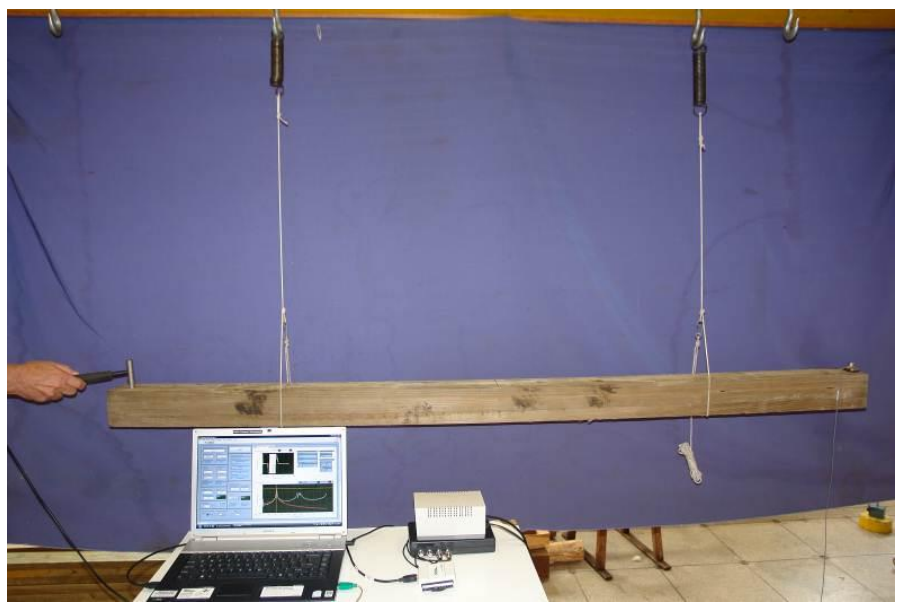

(a)

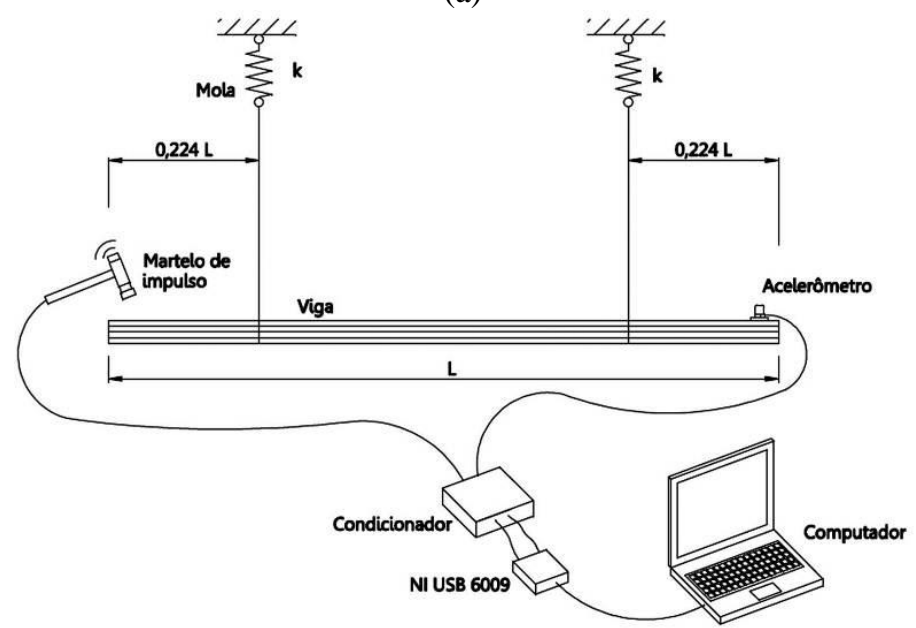

(b)

Figura 3 - Esquema de ensaio de vibração transversal livre

A vibração foi produzida por meio de impactos desferidos verticalmente em uma das extremidades da viga com um martelo de impulso modelo 8206002, fabricado pela Brüel \& Kjær, com sensibilidade igual a $2,3 \mathrm{mV} / \mathrm{N}$. A resposta foi medida por meio de um acelerômetro modelo ISOTRON 7254A-100, fabricado pela Endevco Corporation com sensibilidade igual a $111,6 \mathrm{mV} / \mathrm{g}$, fixado na outra extremidade da viga de MLC com cera de abelha. Os sinais do martelo e do

10 Segundinho, P. G. de A.; Carreira, M. R.; Calil Neto, C.; Regazzi, A. J.; Dias, A. A.; Calil Junior, C. 
acelerômetro foram condicionados por uma placa condicionadora (CARREIRA et al., 2012). O sinal resultante foi digitalizado por uma placa de aquisição modelo NI USB 6009, fabricada pela National Instruments. Para cada impacto desferido foram amostradas 65.536 leituras a uma taxa de amostragem de $10 \mathrm{kHz}$. O tempo necessário para ensaiar cada viga variou de $4 \mathrm{~min}$ a $6 \mathrm{~min}$.

Os sinais foram processados em um programa feito na linguagem de programação Laboratory Virtual Instruments Engineering Workbench (LabVIEW), a partir da qual foi possível obter a Função de Resposta em Frequência (FRF) de cada viga para uma média de 10 espectros. A partir da FRF de acelerância, foi feita a identificação dos parâmetros modais por meio do programa ModalId (CARREIRA et al., 2012), o qual emprega o método Rational Fraction Polynomial (RFP). Na Figura 4 tem-se o exemplo de uma FRF utilizada para a obtenção da frequência do primeiro modo de flexão (parâmetro modal empregado na obtenção do módulo de elasticidade dinâmico).

\section{Análises estatísticas}

Os dados foram analisados estatisticamente, sendo as médias comparadas pelo teste $t$ de Student para dados emparelhados. Foram ajustadas equações de regressão linear simples entre os módulos de elasticidade $E_{\mathrm{M}}$ e $E_{\mathrm{vt}}$. O nível de significância adotado foi de $5 \%$ de probabilidade.

\section{Resultados e discussões}

Nas Tabelas 1 a 3 são apresentados os resultados dos módulos de elasticidade, obtidos por meio dos métodos de ensaio de flexão estática e vibração transversal livre, densidade aparente e frequência natural das peças de MLC. Na Tabela 1 fez-se a comparação dos módulos de elasticidade estático $E_{\mathrm{M}}$ e dinâmico $E_{\mathrm{vt}}$, antes e depois do tratamento preservativo.

O módulo de elasticidade obtido por meio do ensaio de vibração transversal livre $E_{\mathrm{vt}}$ é, em média, $10,46 \%$ maior do que o módulo de elasticidade calculado por meio do ensaio de flexão estática $E_{\mathrm{M}}$, antes do tratamento preservante (Tabela 1). Após o tratamento preservante, o módulo de elasticidade calculado por meio do ensaio de vibração transversal livre $E_{\mathrm{vt}}$ é, em média, $15,14 \%$ maior do que o módulo de elasticidade calculado por meio do ensaio de flexão estática $E_{\mathrm{M}}$ (Tabela 1). Tal comportamento dessas propriedades confirma o que os autores Mochan, Connolly e Moore (2009) já haviam afirmado, ou seja, os ensaios dinâmicos apresentam valores de módulo de elasticidade maiores que os ensaios de flexão estática.

A partir dos valores da Tabela 1, pode-se concluir, para um nível de confiança de $95 \%$, para todas as peças de MLC analisadas nas duas situações, antes e depois do tratamento preservativo, que os valores médios de $E_{\mathrm{vt}}$ não são equivalentes para todas as peças de MLC, o mesmo ocorrendo para os valores de $E_{\mathrm{M}}$, nas duas situações, antes e depois do tratamento preservativo.

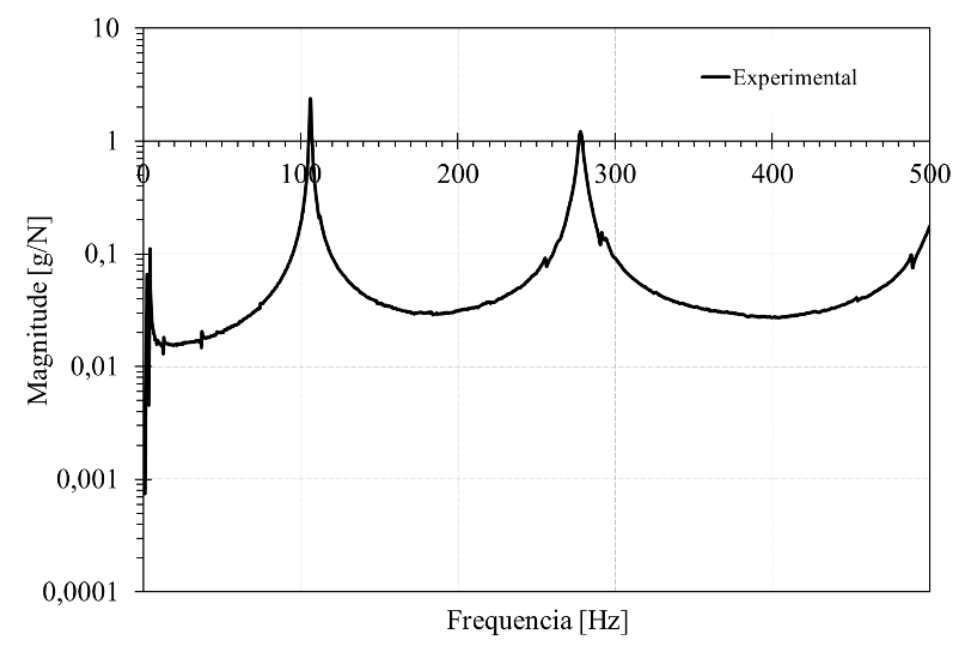

Figura 4 - Exemplo de FRF de uma peça de MLC 
Tabela 1 - Valores médios e coeficientes de variação (\% - entre parênteses) dos módulos de elasticidade estático $E_{M}$ e dinâmico $E_{\mathrm{vt}}$ das peças de $M L C^{* *}$

\begin{tabular}{c|c|c|c}
\hline \multirow{2}{*}{ Madeira } & \multirow{2}{*}{ Tratamento } & \multicolumn{2}{|c}{ Métodos de ensaio } \\
\cline { 3 - 4 } & & $\boldsymbol{E}_{\mathbf{M}}(\mathbf{M P a})$ & $\boldsymbol{E}_{\mathbf{v t}}(\mathbf{M P a})$ \\
\hline Pinus oocarpa & $\mathrm{S}$ & $12.643 \mathrm{aB}(15,40)$ & $13.971 \mathrm{aA}(17,93)$ \\
(PUR) & $\mathrm{C}$ & $12.178 \mathrm{bB}(14,06)$ & $14.044 \mathrm{aA}(17,08)$ \\
\hline Pinus oocarpa $^{*}$ (RFF) & $\mathrm{S}$ & $11.188 \mathrm{aB}(25,86)$ & $12.362 \mathrm{aA}(24,83)$ \\
\hline Lyptus ${ }^{\circledR}$ & $\mathrm{C}$ & $10.727 \mathrm{bB}(24,93)$ & $12.194 \mathrm{aA}(23,69)$ \\
(PUR) $_{\text {Lyptus }}^{\circledR}$ & $\mathrm{S}$ & $18.092 \mathrm{aB}(10,62)$ & $18.679 \mathrm{aA}(10,94)$ \\
(RFF) $_{\text {Teca }}^{\mathrm{C}}$ & $17.266 \mathrm{bB}(10,51)$ & $17.767 \mathrm{bA}(10,62)$ \\
\hline (PUR) & $\mathrm{S}$ & $18.621 \mathrm{aB}(10,31)$ & $19.523 \mathrm{aA}(10,49)$ \\
\hline Teca & $\mathrm{C}$ & $17.536 \mathrm{bB}(9,97)$ & $18.269 \mathrm{bA}(9,72)$ \\
(RFF) & $\mathrm{S}$ & $9.596 \mathrm{aB}(6,09)$ & $10.518 \mathrm{bA}(6,41)$ \\
\hline
\end{tabular}

Nota: **S: sem tratamento preservativo;

C: com tratamento preservativo;

Dentro de cada madeira e cada método de ensaio, a > b pelo teste $t$ de Student ao nível de $5 \%$ de probabilidade; e Dentro de cada madeira e cada tratamento, A > B pelo teste $t$ de Student ao nível de $5 \%$ de probabilidade.

Na Tabela 2 são apresentados os resultados das densidades aparentes e da frequência natural de vibração das peças de MLC.

As densidades aparentes foram maiores após o tratamento preservativo, uma vez que há o acréscimo de massa (Tabela 2). Para um nível de 95\% de confiança, nem todas as peças de MLC analisadas nas duas situações, antes e depois do tratamento preservativo, apresentaram valores médios equivalentes, e as peças de MLC de Pinus oocarpa (RFF) apresentaram a maior variação de densidade. Quanto às frequências naturais apenas em dois casos Pinus oocarpa (PUR) e Teca (RFF) apresentaram valores médios homogêneos para um nível de confiança de $95 \%$ nas peças de MLC analisadas nas duas situações, antes e depois do tratamento preservativo. Portanto, confirma-se que o acréscimo de massa devido ao tratamento preservativo pode ser significativo na densidade aparente e na frequência natural.

$\mathrm{Na}$ Tabela 3 são apresentadas as equações de regressão linear ajustadas entre os módulos de elasticidade $E_{\mathrm{M}}$ e $E_{\mathrm{vt}}$ das peças de MLC, indicadas juntamente com o respectivo coeficiente de determinação $r^{2}$.

$\mathrm{O} E_{\mathrm{M}}$ apresentou correlação linear significativa (P $<0,05)$ com o $E_{\mathrm{vt}}$, tanto nas peças de MLC sem tratamento quanto nas peças de MLC com tratamento, com resultados que variam de 0,85 a 0,99 (Tabela 3).

\section{Conclusões}

O ensaio de vibração transversal livre mostrou-se uma técnica precisa e confiável para avaliar a propriedade mecânica de módulo de elasticidade em peças de MLC, pois, a partir dos resultados experimentais, foi possível fazer estimativas do módulo de elasticidade com boa precisão, sendo significativa $(\mathrm{P}<0,05)$, conforme observado para todos os resultados de regressão linear simples.

Os ensaios realizados neste estudo mostram-se viáveis para identificar as diferenças de desempenho existentes entre os módulos de elasticidade dos tipos de peças de MLC, antes e depois do tratamento preservativo, e permitem estabelecer critérios importantes para a seleção delas quando forem comercializadas.

Na previsão do módulo de elasticidade de peças de MLC por meio de vibração transversal livre, os resultados foram maiores do que os obtidos pelo ensaio de flexão estática, mas esses valores não diferiram estatisticamente $(\mathrm{P}>0,05)$ para cinco dos seis tipos de peças de MLC testadas. 
Tabela 2 - Valores médios e coeficientes de variação (\% - entre parênteses) da densidade aparente e frequência natural das peças de $M L C^{* *}$

\begin{tabular}{c|c|c|c}
\hline \multirow{2}{*}{ Madeira } & \multirow{2}{*}{ Tratamento } & \multicolumn{2}{|c}{ Propriedades } \\
\cline { 3 - 4 } & & $\boldsymbol{r}_{\mathrm{ap}}\left(\mathbf{g} / \mathbf{c m}^{\mathbf{3}}\right)$ & $\mathbf{f}(\mathbf{H z})$ \\
\hline Ponus oocarpa & $\mathrm{S}$ & $596,06 \mathrm{~b}(9,30)$ & $114,84 \mathrm{a}(6,03)$ \\
(PUR) & $\mathrm{C}$ & $621,56 \mathrm{a}(7,69)$ & $114,13 \mathrm{a}(6,39)$ \\
\hline Pinus oocarpa $^{*}$ (RFF) & $\mathrm{S}$ & $573,66 \mathrm{~b}(3,32)$ & $109,80 \mathrm{a}(10,56)$ \\
\hline Lyptus $^{\circledR}$ & $\mathrm{C}$ & $591,76 \mathrm{a}(3,72)$ & $108,43 \mathrm{~b}(10,56)$ \\
\hline (PUR) & $\mathrm{S}$ & $719,46 \mathrm{a}(2,79)$ & $116,55 \mathrm{a}(4,79)$ \\
Lyptus ${ }^{\circledR}$ & $\mathrm{C}$ & $720,32 \mathrm{a}(3,11)$ & $114,91 \mathrm{~b}(4,61)$ \\
(RFF) $_{\text {Teca }}$ & $\mathrm{S}$ & $783,87 \mathrm{a}(5,38)$ & $113,08 \mathrm{a}(4,68)$ \\
(PUR) & $\mathrm{C}$ & $785,16 \mathrm{a}(5,39)$ & $111,14 \mathrm{~b}(4,88)$ \\
\hline Teca & $\mathrm{S}$ & $577,18 \mathrm{~b}(4,14)$ & $107,09 \mathrm{~b}(3,66)$ \\
(RFF) & $\mathrm{C}$ & $581,48 \mathrm{a}(4,17)$ & $107,61 \mathrm{a}(3,59)$ \\
\hline
\end{tabular}

Nota: **S: sem tratamento preservativo;

C: com tratamento preservativo; e

Dentro de cada madeira e cada propriedade, $a>b$ pelo teste $t$ de Student ao nível de $5 \%$ de probabilidade.

Tabela 3 - Equações de regressão linear ajustadas $(\hat{\mathbf{y}}=\mathrm{ax}+\mathrm{b})$ entre os módulos de elasticidade $E_{M}$ e $E_{\mathrm{vt}}$ das peças de MLC

\begin{tabular}{c|c|c|c|c|c|c|c}
\hline Espécies & Tratamento & $\hat{\mathbf{y}}(\mathbf{M P a})$ & $\mathbf{x}(\mathbf{M P a})$ & $\mathbf{a}$ & $\mathbf{b}(\mathbf{M P a})$ & $\mathbf{r}$ & $\mathbf{r}^{2}$ \\
\hline Pinus oocarpa & $\mathrm{S}$ & $E_{\mathrm{M}}$ & $E_{\mathrm{vt}}$ & $0,77^{*}$ & $1914,05^{*}$ & $0,99^{*}$ & $0,98^{*}$ \\
(PUR) & $\mathrm{C}$ & $\mathrm{E}_{\mathrm{M}}$ & $\mathrm{E}_{\mathrm{vt}}$ & $0,71^{*}$ & $2227,60^{*}$ & $0,99^{*}$ & $0,99^{*}$ \\
\hline Pinus oocarpa $^{\mathrm{n}}$ & $\mathrm{S}$ & $E_{\mathrm{M}}$ & $E_{\mathrm{vt}}$ & $0,92^{*}$ & $-246,64^{\mathrm{ns}}$ & $0,98^{*}$ & $0,96^{*}$ \\
(RFF) & $\mathrm{C}$ & $E_{\mathrm{M}}$ & $E_{\mathrm{vt}}$ & $0,91^{*}$ & $-390,64^{\mathrm{ns}}$ & $0,98^{*}$ & $0,97^{*}$ \\
\hline Lyptus $^{\circledR}$ & $\mathrm{S}$ & $E_{\mathrm{M}}$ & $E_{\mathrm{vt}}$ & $0,89^{*}$ & $1418,75^{\mathrm{ns}}$ & $0,95^{*}$ & $0,90^{*}$ \\
(PUR) & $\mathrm{C}$ & $E_{\mathrm{M}}$ & $E_{\mathrm{vt}}$ & $0,93^{*}$ & $769,44^{\mathrm{ns}}$ & $0,96^{*}$ & $0,93^{*}$ \\
\hline Lyptus & $\mathrm{S}$ & $E_{\mathrm{M}}$ & $E_{\mathrm{vt}}$ & $0,92^{*}$ & $684,36^{\mathrm{ns}}$ & $0,98^{*}$ & $0,96^{*}$ \\
(RFF) & $\mathrm{C}$ & $E_{\mathrm{M}}$ & $E_{\mathrm{vt}}$ & $0,96^{*}$ & $47,63^{\mathrm{ns}}$ & $0,97^{*}$ & $0,95^{*}$ \\
\hline Teca & $\mathrm{S}$ & $E_{\mathrm{M}}$ & $E_{\mathrm{vt}}$ & $0,78^{*}$ & $1423,76^{\mathrm{ns}}$ & $0,89^{*}$ & $0,80^{*}$ \\
(PUR) & $\mathrm{C}$ & $E_{\mathrm{M}}$ & $E_{\mathrm{vt}}$ & $0,72^{*}$ & $1862,97^{\mathrm{ns}}$ & $0,88^{*}$ & $0,78^{*}$ \\
\hline Teca & $\mathrm{S}$ & $E_{\mathrm{M}}$ & $E_{\mathrm{vt}}$ & $0,41^{*}$ & $5020,92^{*}$ & $0,85^{*}$ & $0,73^{*}$ \\
(RFF) & $\mathrm{C}$ & $E_{\mathrm{M}}$ & $E_{\mathrm{vt}}$ & $0,93^{*}$ & $-271,06^{\mathrm{ns}}$ & $0,97^{*}$ & $0,94^{*}$ \\
\hline
\end{tabular}

Nota: * - significativo ao nível de $5 \%$ de probabilidade pelo teste t de Student $(P<0,05)$; e

ns - não significativo $(P>0,05)$.

A partir da observação e manuseio das peças de MLC realizados durante o ensaio de vibração transversal livre, pode-se concluir que é um método prático para avaliação do módulo de elasticidade de elementos estruturais de MLC, sendo uma alternativa viável aos equipamentos de grandes dimensões e elevado custo utilizados no ensaio de flexão estática. Dessa forma, acredita-se que é possível o controle de qualidade, com menores custos, desses produtos derivados de madeira produzidos pela indústria. Mesmo não sendo quantificado, apenas observado, constatouse também como característica positiva no ensaio de vibração transversal livre o tempo, que foi inferior ao despendido no ensaio de flexão estática.

\section{Referências}

AMERICAN SOCIETY FOR TESTING AND MATERIALS. ASTM D198: standard test methods of static tests of lumber in structural sizes. Philadelphia, 1997. 26 p.

\section{ASSOCIAÇÃO BRASILEIRA DE NORMAS}

TÉCNICAS. NBR 8458: cruzetas de madeira para redes de distribuição de energia elétrica: especificações. Rio de Janeiro, 2010. 11 p.

\section{ASSOCIAÇÃO BRASILEIRA DE NORMAS}

TÉCNICAS. NBR 7190: projetos de estruturas de madeira. Rio de Janeiro, 1997. 107 p. 
BODIG, J.; JAYNE, B. A. Mechanics of Wood and Wood Composites. New York: V. N. Reinhold, 1993.

CANADIAN STANDARDS ASSOCIATION.

CAN/CSA 0177: qualification code for manufactures of structural glued: laminated timber. Alberta, 2006. 56 p.

CARREIRA, M. R. et al. Bending Stiffness Evaluation of Teca and Guajará Lumber Through Tests of Transverse and Longitudinal Vibration. Acta Scientiarum. Technology, Maringá, v. 34, n. 1, p. 27-32, jan./mar. 2012.

CARREIRA, M. R. Avaliação da Rigidez à Flexão de Toras de Madeira por meio de Vibração Transversal. 382 f. São Carlos, 2012. Tese (Doutorado em Engenharia de Estruturas) Escola de Engenharia de São Carlos, Universidade de São Paulo, São Carlos, 2012.

FOREST PRODUCTS LABORATORY. Wood

Handbook: wood as an engineering material. Madison: U.S. Department of Agriculture, 1999. $463 \mathrm{p}$.
MCCONNELL, K. G.; VAROTO, P. S. Vibration Testing: theory and practice. 2. ed. Hoboken: John Wiley \& Sons, 2008. 652 p.

MOCHAN, S.; CONNOLLY, T.; MOORE, J. Using Acoustic Tools in Forestry and the Wood Supply Chain. Edinburgh: Forestry Commission, sep. 2009. Technical Note. 6 p.

\section{ROSS, R. J.; PELLERIN, R. F. Nondestructive}

Testing For Assessing Wood Members in

Structure: a review. Madison: United State, Department of Agriculture, 1994. General Technical Report FPL-GTR-70.

YANG, T. H. et al. Evaluation of the Mechanical Properties of Douglas-Fir and Japanese Cedar Lumber and Its Structural Glulam by Nondestructive Techniques. Construction and Building Materials, Amsterdam, v. 22, p. 487 493, 2008.

Revista Ambiente Construído

Associação Nacional de Tecnologia do Ambiente Construído

Av. Osvaldo Aranha, $99-3^{\circ}$ andar, Centro

Porto Alegre - RS - Brasil

CEP $90035-190$

Telefone: +55 (51) 3308-4084

Fax: +55 (51) 3308-4054

www.seer.ufrgs.br/ambienteconstruido

E-mail: ambienteconstruido@ufrgs.br

14 Segundinho, P. G. de A.; Carreira, M. R.; Calil Neto, C.; Regazzi, A. J.; Dias, A. A.; Calil Junior, C. 